\title{
Prospects of the high luminosity LHC from ATLAS and CMS
}

\author{
Alessia Tricomi ${ }^{* \dagger}$ \\ University of Catania and INFN Catania, Italy \\ E-mail: alessia.tricomideern.ch
}

The physics accessible at the high-luminosity phase of the LHC extends well beyond that of the earlier LHC program. This paper presents few highlights to illustrate the substantially enhanced reach in the field of Higgs physics and SUSY searches achievable with an increased integrated luminosity of $3000 \mathrm{fb}^{-1}$, which motivates the planned upgrades of the ATLAS and CMS detector.

The European Physical Society Conference on High Energy Physics

22-29 July 2015

Vienna, Austria

*Speaker.

†n behalf of the CMS and ATLAS Collaborations 


\section{Introduction}

The 7 and $8 \mathrm{TeV}$ data taking in 2010-2012, the so-called Run1, at LHC was very successfull for both ATLAS and CMS experiments. The LHC machine has delivered an integrated luminosity of about $30 \mathrm{fb}^{-1}$ to each of the two general purpose experiments and the collected data have yielded a huge amount of interesting Physics results, which culminated in the discovery of a Higgslike boson [1,2] in 2012. While it represents a milestone in understanding the origin of elementary particle mass, several fundamental questions in particle Physics, like the origin of Dark Matter, the asymmetry between matter and anti-matter, the force unification, are still far from being explained, hence the results obtained in Run1 have further motivated the search for new physics and the need of precision study of the discovered Higgs-like particle. The increase in energy during the ongoing Run2 is certainly the starting point to address this program, however it may not be enough to address the New Physics quests and the increase in luminosity, foreseen at HL-LHC, will complement and further enlarge the Physics potential at LHC adding the possibility to explore rare phenomena and increasing the phase space coverage. In this document, which by far cannot be considered as an exhaustive resume of the HL-LHC Physics potential, few highlights of the ongoing studies in preparation towards the HL phase are discussed.

\section{The HL-LHC}

After the successfull data-taking in 2010-2012 and the "Long Shutdown 1" period from 2013 till mid of 2015 (LS1), the LHC machine and its experiments are now entered in the so-called Run2 period. During the first period of Run2 it is expected to operate at the original perfomance goal of LHC with a peak luminosity of $10^{34} \mathrm{~cm}^{-2} \mathrm{~s}^{-1}$ and $25 \mathrm{~ns}$ bunch spacing, resulting in an average number of pp interactions per bunch crossing, $\mu$, of approximately 25 (referred to as "pile-up"). Subsequentely, upgrades to the LHC machine during the "Long Shutdown 2" in mid-2018-2019 will increase the instantaneous luminosity to above $2 \times 10^{34} \mathrm{~cm}^{-2} \mathrm{~s}^{-1}$, with the pile-up increasing to $\mu \sim 60$, providing an integrated luminosity of $300 \mathrm{fb}^{-1}$ by 2023 . To maintain the present performance in this period, the ATLAS [3] and CMS [4] detector will undergo an initial series of staged upgrades in the period from LS1 through LS2, known as the Phase-I Upgrade.

By 2023, during the LS3, the quadrupoles that focus the beams at the ATLAS and CMS collision regions will be replaced and crab-cavities will be added to optimize the bunch overlap at each interaction region. These changes will produce a significant increase in the LHC delivered luminosity. The high luminosity period that follows LS3 is referred as HL-LHC or Phase-II. The proposed operating scenario is to level the instantaneous luminosity at $5 \times 10^{34} \mathrm{~cm}^{-2} \mathrm{~s}^{-1}$ and to deliver about $300 \mathrm{fb}^{-1}$ per year for a further 10 years of operation. Under these conditions the event pile-up will rise substantially ( $\mu \sim 140-200$ ) to become a major challenge for the experiments, and the performance degradation due to integrated radiation dose will need to be addressed. A full upgrade program is hence envisaged with the basic goal to maintain the same excellent performance of the ATLAS and CMS detectors in terms of efficiency, resolution, and background rejection for all the physics objects as in the current run. Despite different technological choices will be adopted by the two collaborations, the basic strategy is common and foresees:

- the replacement of the Tracker; 
- the replacement/upgrade of the calorimeter and muon systems;

- the upgrade of the L1 trigger to include track information.

The Phase-II subdetectors should have higher granularity to cope with the increased pile-up, higher radiation resistance and extended coverage to increase acceptance and improve performances. Details of the detector upgrades can be found elsewhere in these Proceedings [5].

\section{The Physics prospect at HL-LHC}

The Physics program at the HL-LHC is very broad and will build on the experience acquired, and the results obtained, in the first phase of the LHC operation, with more than $300 \mathrm{fb}^{-1}$ of accumulated data. The main goal is to continue the quest to answer fundamental questions in particle physics, on one hand with precision measurements, especially of the Higgs-like sector, including detailed study of any new phenomena discovered before LS3; and on the other hand by direct searches for new physics.

Both ATLAS and CMS are already studying the sensitivity to different channels addressing the new features foreseen for the upgraded detectors. For doing that both experiments have used full simulation studies of the new envisaged detectors in order to extract a parameterization of the physics object performances. The parameterised response functions account for the effect of the pile-up ranging between 50 and 140 with $25 \mathrm{~ns}$ bunch spacing and are injected either in generator level studies or in fast simulation tools $[6,7,8,9]$. In some cases, extrapolation from Run1 have been used. In addition CMS has also taken into account the effect of the aging of the detector.

\subsection{Higgs sector}

The measurements of Higgs boson properties made with the Run1 data are already impressive, however the HL-LHC with its 10 times more deliverable luminosity wrt to the expectation for Run2/Run3 will enable a full set of Higgs studies comprising precision measurements of many of the properties of the Higgs boson, such as its couplings, a detailed CP analysis of the Higgs boson as well the measurement of rare Standard Model (SM) and Beyond the Standard Model (BSM) decays. Figure 1 shows the estimated precision on the measurements for modified couplings for a SM-like Higgs boson expected by CMS. Two scenarios are used to extrapolate uncertainties, namely scenario 1 where all systematic uncertainties are left unchanged, and scenario 2 where the theoretical uncertainties are reduced by a factor of two and all other systematic uncertainties are reduced by the square root of the integrated luminosity. The two scenarios are expected to bracket a realistic extrapolation. Similar results are also reported by ATLAS [10]. Compared to a precision of about $20 \%$ on Higgs boson couplings today, percent-level precision can be reached for most coupling measurements.

In particular, the coupling to the second-generation fermions will be probed for the first time measuring the Higgs boson decay to two muons. Higgs boson coupling to charged leptons is a crucial measurement. The coupling to electrons is too small to measure, but the coupling to $\tau$ leptons will be well-measured by the end of Phase-II. The couplings to the muons, however, whose branching fraction is only $\sim 10^{-4}$ will become accessible only at the HL-LHC. Projections of the analysis to the HL-LHC era show that the coupling of the Higgs boson to muons can be measured 


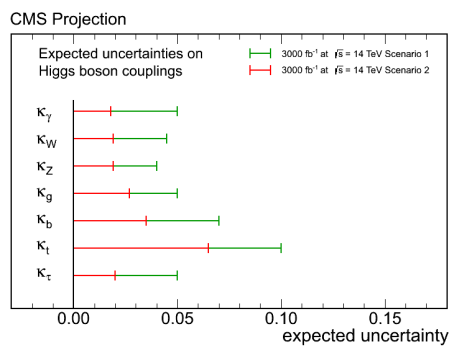

Figure 1: Estimated precision on the measurements for modified couplings for a SM-like Higgs boson for and integrated luminosity of $3000 \mathrm{fb}^{-1}$ for the two scenarios described in the text [9].

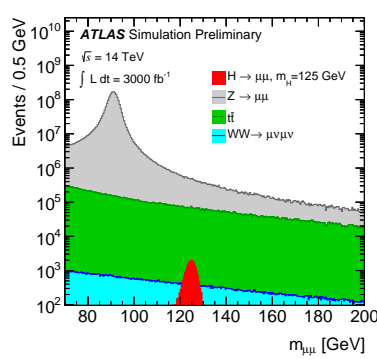

Figure 2: Distribution of the estimated $\mu^{+} \mu^{-}$invariant mass of the $H \rightarrow \mu^{+} \mu^{-}$signal and background processes at HL-LHC [10].

with a precision of about $8 \%$, still limited by the statistical uncertainty. In $H \rightarrow \mu^{+} \mu^{-}$events the kinematics of the Higgs boson can be fully reconstructed and this channel can contribute to mass measurements due to the high resolution of the reconstructed $\mu^{+} \mu^{-}$invariant mass. Searches in this channel are challenged by the low branching fraction and high contributions of background processes such as $Z / \gamma^{*}$ production. The signal will consist of a small bump over a large di-muon background from Drell-Yan events, hence an excellent di-muon mass resolution is crucial. The resulting $\mu^{+} \mu^{-}$invariant mass distribution of the signal and background processes expected by the ATLAS detector for an integrated luminosity of $3000 \mathrm{fb}^{-1}$ are shown in Fig. 2.

The measurement of the Higgs boson coupling to muons is expected to improve with the squareroot of the improvement in resolution and efficiency. A crucial role in the study of this channel is hence played by the performance of the new tracking system. CMS foresees a $40 \%$ better mass resolution and a $20 \%$ larger efficiency to reconstruct the muon pair, thanks to the reduction of material and better spatial measurements of the upgraded Phase-II tracking detector with respect to an aged Phase-I detector.

The very high integrated luminosity collected at HL-LHC will enable the study of the Higgs potential through the measurement of the Higgs boson pair production. An intrinsic property of the SM Higgs boson is, indeed, its self-coupling, the strength of which must be established in order to test whether the observed Higgs boson fulfills its crucial role in electroweak symmetry breaking. The process is also sensitive to other BSM effects, as new physics can modify the rate of Higgs boson pair production. Di-Higgs events can be produced via a box diagram and through the Higgs boson self-coupling contribution. The two processes interfere destructively and the cross section is near minimum for the SM. The HL-LHC is hence mandatory to study these processes. Both ATLAS [11] and CMS [9] have performed studies of the di-Higgs production and decay into $b b \gamma \gamma$ which offers the potential for a clean HH signal extraction thanks to the narrow mass peak of the $H \rightarrow \gamma \gamma$ decay, as shown in Fig. 3. About 8-9 signal events are expected by each of the two experiments after trigger and event selections corresponding to a signal significance of $\sim 1.3 \sigma$ per experiment for the SM scenario.

In addition, CMS has also studied the $b b W W$ channel, where the $\mathrm{W}$ boson decays leptonically, and $b b \tau_{h} \tau_{h}$ and bb $\tau_{h} \tau_{\mu}$ final states, where $\tau_{h}$ denotes hadronic tau decays, and $\tau_{\mu}$ denotes tau decays to muons. About $9000 b b \tau \tau$ di-Higgs boson events per experiment are expected at HL-LHC with 3 
$\mathrm{ab}^{-1}$. However, the t⿱亠t background with fully leptonic decays to taus is overwhelming. The use of kinematic variables as well as Boost Decision Tree (BDT) technique seems to be proficient in the discrimination of the signal and background contribution, as shown in Fig. 4. A combined signal significance of $0.9 \sigma$ is expected in the $\tau \tau$ final states.

Combining the studies of $b b \gamma \gamma$ and $b b \tau \tau$ final states, the expected significance for the di-Higgs boson production is about 1.9 standard deviations with an expected uncertainty in the signal yield of approximately 54\%. Significant improvements in future studies of the di-Higgs productions are expected by combining multiple channels and using results from ATLAS and CMS.
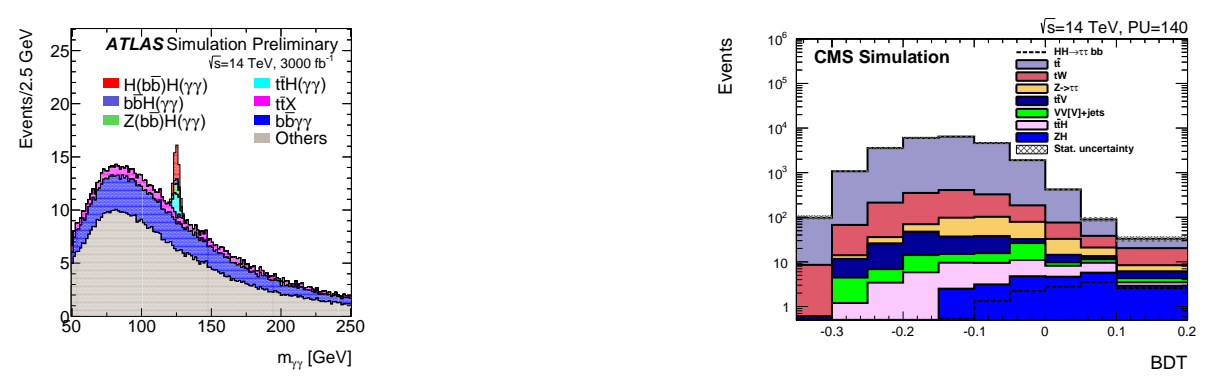

Figure 3: Invariant mass distributions of $\mathrm{m}_{\gamma \gamma}$ for Figure 4: Distribution of the BDT discriminant vari$3000 \mathrm{fb}^{-1}$ for $H H \rightarrow b b \gamma \gamma$ signal and background able for $H H \rightarrow b b \tau_{h} \tau_{h}$ signal and background events events after applying all the selection criteria de- (Ref. [9]) for $3000 \mathrm{fb}^{-1}$.

scribed in Ref. [11].

\subsection{SUSY searches}

The discovery (or exclusion) of weak-scale SUSY is one of the highest physics priorities for the current and future LHC, including the high luminosity upgrade, HL-LHC. Despite the lack of evidence in Run1, the motivations for supersymmetry are strong: SUSY provides a candidate particle, the lightest supersymmetric particle (LSP), that may account for all or part of the astrophysical dark matter; second, if a SUSY particle spectrum is present, the three running gauge couplings of the SM interactions can converge at a common high energy, a requirement for gauge unification. Finally, the discovery of a Higgs-like boson by the ATLAS and CMS experiments has given new urgency to the gauge hierarchy problem. Indeed, a broad class of SUSY scenarios, known as natural models, can stabilize the Higgs boson mass through additional contributions involving diagrams with the scalar superpartner, $\tilde{t}$, of the top quark (stop), as well as other SUSY particles.

In order to assess the potentiality to discover and study SUSY at HL-LHC and the impact of the upgraded detectors, ATLAS has used a set of simplified SUSY models together with a set of benchmark scenarios [12], while CMS has used both simplified models as well as five fullspectrum benchmark models, which include three natural SUSY scenarios, as well as stau and stop coannihilation scenarios and has used a signature-based search strategy. Figure 5 gives an overview of the CMS search strategy and shows the discovery significances expected at the end of Run3 and at the HL-LHC for the five full-spectrum benchmark models considered. Details of the analyses can be found in Ref. [13].

The factor 10 increase in integrated luminosity expected at HL-LHC will play a central role to significantly extend the discovery reach not only for squarks and gluinos, which have the highest 


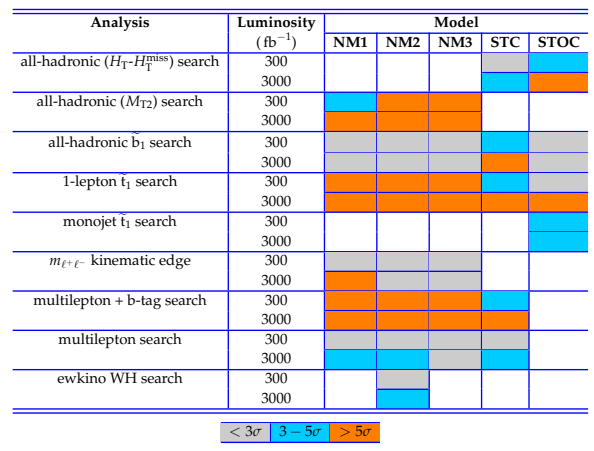

Figure 5: Overview of the CMS SUSY search analysis strategy.

production cross-section at LHC, but also in the electroweak sector.

Chargino-neutralino production has been studied in the $\mathrm{WZ}$ and $\mathrm{Wh}$ mediated scenario, in which the $\mathrm{Z}$ decays leptonically thus originating a 3-lepton final states, while the Higgs bosons can decay in leptons, taus or bb. A $5 \sigma$ discovery reach up to $850-950 \mathrm{GeV}$ in $\tilde{\chi}_{1}^{ \pm}, \tilde{\chi}_{2}^{0}$ mass can be achieved at HL-LHC in both WZ-Wh mediated scenarios depending on the decay channel considered. Recent studies [14] have also demonstrated that the use of a multivariate analysis (MVA) technique wrt standard cut-and-count analysis can significantly improve by about $20 \%$ the $5 \sigma$ discovery reach and exclusion limits (Fig. 6).

The degradation of the detector performance as the result of radiation damage will affect most observables relevant to SUSY searches. The effect of the degradation in the trigger efficiency, lepton identification, b-tagging efficiency, and missing transverse energy (MET) resolution have been estimated for this channel, and the key results are summarized in Fig. 7. This search is particularly sensitive to detector performances and the gain in discovery reach is significantly reduced if the existing aged detectors are used thus demonstrating the importance of the foreseen upgrades.

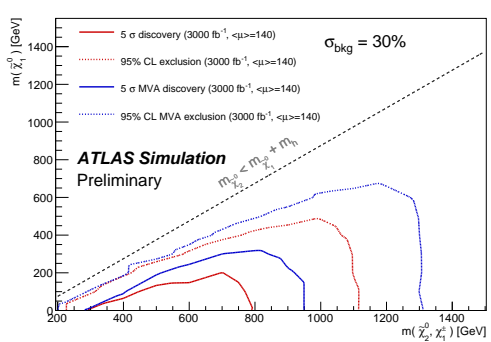

Figure 6: Expected 95\% exclusion and discovery contours for the $3000 \mathrm{fb}^{-1}$ luminosity scenario in the $m\left(\tilde{\chi}_{1}^{0}\right), m\left(\tilde{\chi}_{1}^{ \pm}, \tilde{\chi}_{2}^{0}\right)$ plane for the Wh-mediated simplified model, comparing a cut and count and a MVA approaches [14].

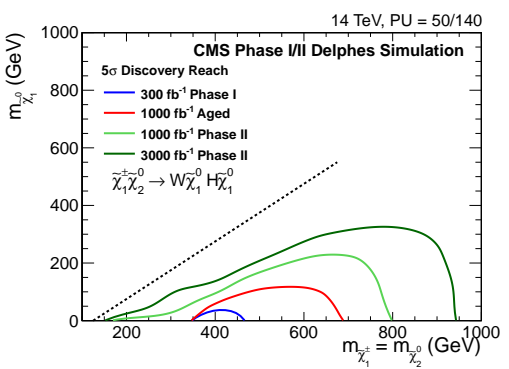

Figure 7: Effect of detector aging on the sensitivity for the SUSY search for $\tilde{\chi}_{1}^{ \pm}, \tilde{\chi}_{2}^{0}$ in the Wh final states. The estimated uncertainties in detector performance for trigger efficiency, lepton identification, b-tagging efficiency, and MET resolution are applied [9].

Significant improvement in the discovery reach can be achieved also for gluino and squark production. A $5 \sigma$ discovery reach up to $\sim 2.5$ and $\sim 3 \mathrm{TeV}$ can be achieved for gluinos and squarks, respectively.

In addition, naturalness arguments require that the third generation squarks, sbottom and stop, 
have mass in the range of $1 \mathrm{TeV}$, thus the discovery at LHC will be possible, in one or more of the favorable search signatures already with $300 \mathrm{fb}^{-1}$. At HL-LHC, with $3000 \mathrm{fb}^{-1}$, sensitivity to other key signatures with heavier stop and sbottom can be achieved and going one step further, in case of discovery, the properties of the discovered SUSY particles can be measured.

\section{Conclusions}

The HL-LHC foreseen to start in about ten years from now will offer a unique opportunity to deeply explore the Higgs sector and will represent an excellent probe for high scale New Physics. The expected $3000 \mathrm{fb}^{-1}$ dataset at $14 \mathrm{TeV}$ will allow large gains in precision, discovery potential, and will make a number of important, low cross section measurements possible. The detector upgrades foreseen by ATLAS and CMS will ensure optimal performances despite the very hostile environment. Work is ongoing to be ready and well prepared for this new exciting LHC-era.

\section{References}

[1] ATLAS Collaboration, Observation of a new particle in the search for the Standard Model Higgs boson with the ATLAS detector at the LHC, Phys. Lett. B 716 (2012) 1.

[2] CMS Collaboration, Observation of a new boson at a mass of $125 \mathrm{GeV}$ with the CMS experiment at the LHC, Phys. Lett. B 716 (2012) 30.

[3] ATLAS Collaboration, The ATLAS Experiment at the CERN Large Hadron Collider, JINST 3 S08003.

[4] CMS Collaboration, The CMS Experiment at the CERN LHC, JINST 3 S08004.

[5] D.C. Contardo, Detector R\&D for the HL-LHC upgrade, (EPS-HEP 2015) 018.

[6] ATLAS Collaboration, Performance assumptions based on full simulation for an upgraded ATLAS detector at a High-Luminosity LHC, ATL-PHYS-PUB-2013-009.

[7] ATLAS Collaboration, Performance assumptions for an upgraded ATLAS detector at a High-Luminosity LHC, ATL-PHYS-PUB-2013-004.

[8] J. de Favereau et al., DELPHES 3, A modular framework for fast simulation of a generic collider experiment, arXiv:1307.6346.

[9] CMS Collaboration, Technical Proposal for the Phase-II Upgrade of the Compact Muon Solenoid, CERN-LHCC-2015-010.

[10] ATLAS Collaboration, Projections for measurements of Higgs boson signal strengths and coupling parameters with the ATLAS detector at the HL-LHC, ATL-PHYS-PUB-2014-016.

[11] ATLAS Collaboration, Prospects for measuring Higgs pair production in the channel $H(\rightarrow \gamma \gamma) H(\rightarrow \mathrm{b} \overline{\mathrm{b}})$ using the ATLAS detector at the HL-LHC, ATL-PHYS-PUB-2014-019.

[12] ATLAS Collaboration, Search for Supersymmetry at the high luminosity LHC with the ATLAS experiment, ATL-PHYS-PUB-2014-010.

[13] CMS Collaboration, Supersymmetry discovery potential in future LHC and HL-LHC running with the CMS detector, CMS Physics Analysis Summary CMS-PAS-SUS-14-012, 2014.

[14] ATLAS Collaboration, Prospect for a search for direct pair production of a chargino and a neutralino decaying via a $W$ boson and the lightest Higgs boson in final states with one lepton, two b-jets and missing transverse momentum at the high luminosity LHC with the ATLAS Detector,

ATL-PHYS-PUB-2015-032. 